\title{
New research in prostate cancer, ASCO 2017
}

\author{
Naveen S. Basappa, MD, FRCPC'; Anil Kapoor, MD, FRCSC 2 \\ 'Cross Cancer Institute \& University of Alberta, Edmonton, AB; ${ }^{2}$ CUAJ Associate Editor \& McMaster University, Hamilton, ON; Canada
}

Cite as: Can Urol Assoc J 2017; 11 (8Supp15):S303-4.

http://dx.doi.org/10.5489/cuaj.4842

\section{New evidence with oral hormonal agents abiraterone acetate and enzalutamide}

The oral agents abiraterone acetate (in combination with prednisone $[\mathrm{AAP}]$ ) and enzalutamide are currently recommended by Canadian guidelines as first-line options for asymptomatic or minimally symptomatic metastatic castration-resistant prostate cancer (mCRPC) among chemotherapy-naïve patients and as an option for patients who progress on or after docetaxel-based chemotherapy. ${ }^{1}$ At ASCO 2017, the results of two studies were presented in which this combination was evaluated earlier in the treatment paradigm: in combination with androgen-deprivation therapy (ADT) among newly diagnosed patients with high-risk, metastatic, castration-naïve prostate cancer (mCNPC)., ${ }^{2,3}$ In addition to the presentations at ASCO, both studies were also simultaneously published in the New England Journal of Medicine. ${ }^{4,5}$

In the LATITUDE study, ${ }^{2,4}$ the definition of high-risk was at least two of the following three characteristics: Gleason score $\geq 8$, presence of $\geq 3$ lesions on bone scan, and presence of measurable visceral lesion.

Patients were randomized to receive ADT + AAP (1000/5 mg QD, $n=597)$ or ADT + placebo $(n=602)$. The treatment arms were well-balanced in terms of baseline characteristics. The co-primary endpoints were overall survival (OS) and radiographic progression-free survival (rPFS).

Over a median followup of 30.4 months, the inclusion of AAP was associated with a significant $38 \%$ reduction in mortality risk (hazard ratio [HR] 0.62; 95\% confidence interval [Cl] 0.51-0.76; Fig. 1A). Median OS was 34.7 months in the ADT-alone arm and not reached in the AAP-containing arm. The three-year OS was $66 \%$ for ADT + AAP and $49 \%$ for ADT + placebo. Subgroup analysis showed that the HR was in favour of the inclusion of AAP across all subgroups. For rPFS, ADT + AAP was associated with a significant $53 \%$ reduction in risk (HR 0.47; 95\% $\mathrm{Cl} 0.39-0.55)$ compared to ADT + placebo (Fig. 1B). Median rPFS was 33.0 months in the ADT + AAP arm and 14.8 months for ADT + placebo. The inclusion of AAP was also associated with favourable results in all secondary endpoints, including time to prostatespecific antigen (PSA) progression, time to pain progression, time to next symptomatic skeletal event, time to chemotherapy, and time to subsequent prostate cancer therapy.

The overall safety profile of ADT + AAP was similar to that seen in trials among patients with $\mathrm{mCRPC}$.

The other trial in this setting presented at ASCO 2017 was the STAMPEDE study, which enrolled 1917 patients with high-risk prostate cancer. ${ }^{3,5}$ For inclusion in this study, patients had to be hormone-naïve and be newly diagnosed with metastatic or node-positive disease, or have high-risk locally advanced disease defined as having at least two of the following: stage T3/4, PSA $>40 \mathrm{ng} / \mathrm{mL}$, and Gleason score $8-10$. Alternatively, patients could be enrolled if they relapsed after previous radical prostatectomy or radiotherapy, and had at least one of the following inclusion criteria: PSA at least $4 \mathrm{ng} / \mathrm{mL}$ and rising, with doubling time less than six months; PSA $20 \mathrm{ng} / \mathrm{mL}$ or greater, node-positive, or metastatic. Patients meeting these criteria were randomized to receive standard of care with $(n=960)$ or without $(n=957)$ AAP. The primary outcome measure was OS.

As shown in Fig. 2A, the addition of AAP to ADT was associated with a $37 \%$ reduction in mortality (HR 0.63; $95 \%$ $\mathrm{CI} 0.52-0.76$ ). A significant $71 \%$ improvement in failure-free survival was also observed (HR 0.29; 95\% Cl 0.25-0.34; Fig. 2B). Significant improvements were also observed favouring AAP for skeletal related outcomes (HR 0.46; 95\% $\mathrm{Cl}$ 0.37-0.58). Overall, AAP was well-tolerated in this study.

Previous data from the CHAARTED study demonstrated benefit of docetaxel with ADT in metastatic hormone-sensitive prostate cancer. Both the LATITUDE and STAMPEDE studies demonstrated that the addition of AAP to ADT in metastatic hormone-sensitive prostate cancer provides significant benefit to patients over ADT alone. Now, with these new data, we have a number of options to help our patients in this space. Questions regarding the benefit of combining docetaxel, AAP, and ADT remain to be answered.

In the $\mathrm{mCRPC}$ setting, investigators presented the results of a crossover study of AAP vs. enzalutamide. ${ }^{6}$ A total of 200 patients with treatment-naïve mCRPC were randomized to AAP (at a dose of 1000/10 mg daily) or enzalutamide (160 mg daily) and were crossed over to the other therapy at progression. The primary objective was to compare response and time to PSA progression (TTPP) after 


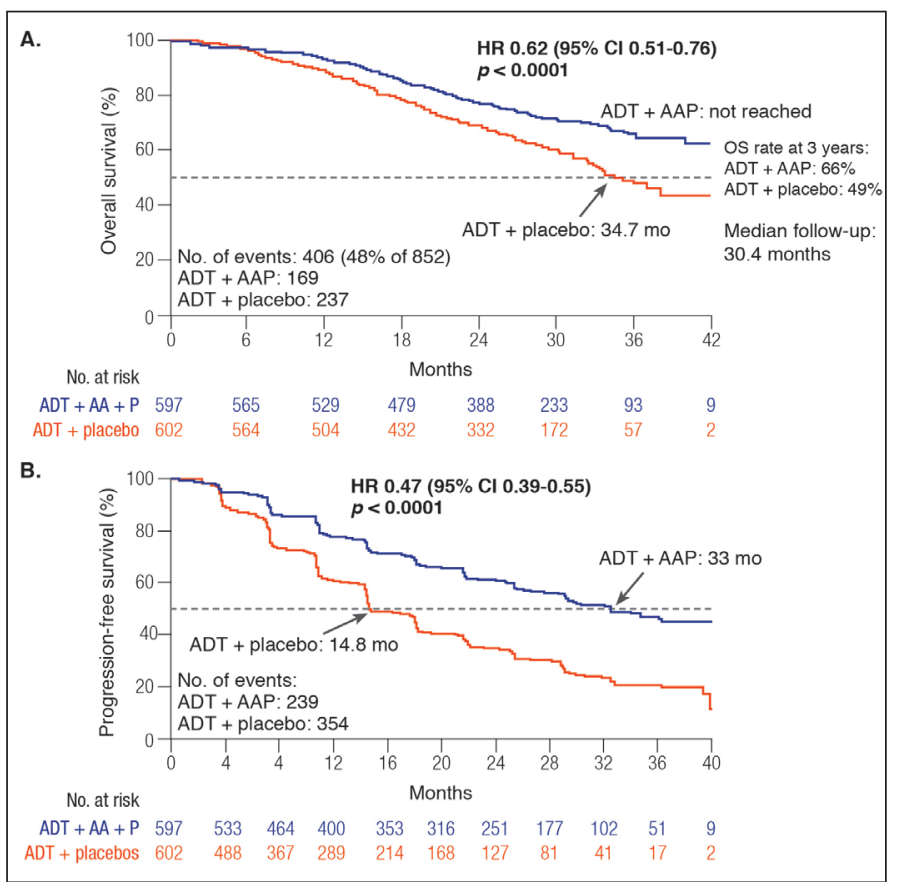

Fig. 1. (A) Overall survival (OS); and (B) radiographic progression-free survival for abiraterone acetate $(A A)+$ prednisone $(P)$ vs. placebo (in addition to androgen-deprivation therapy [ADT]) in metastatic, castration-naïve prostate cancer (LATITUDE). Cl: confidence interval.

second-line therapy. Secondary endpoints were reported at this meeting, including PSA $\geq 50 \%$ decline (PSA50) from baseline, TTPP with first-line therapy, and correlation with deep targeted sequencing of $73 \mathrm{mCRPC}$ genes in circulating tumour DNA (ctDNA).

Results showed that, although the enzalutamide arm demonstrated a more substantial PSA response at 12 weeks (77\% compared to $55 \%$ ), there were no significant differences between arms with respect to TTPP in the first-line setting (AAP 7.4 months vs. enzalutamide 8.0 months; HR 0.88; 95\% $\mathrm{Cl}$ 0.61-1.27). Furthermore, sequencing results demonstrated that patients with pathogenic ctDNA changes (i.e., TP53 and BRCA2/ATM alterations) had poorer outcomes.

Analyses from this comparison study of AAP vs. enzalutamide examining quality of life, cognition and depression were also presented at ASCO 2017.7 The FACT-P quality of life (QoL) questionnaire, patient health questionnaire (PHQ-9 depression test), and Montreal Cognitive Assessment (MoCA) were completed as a part of this study. A total of 145 baseline and 142 followup assessments out of a possible 202 were completed. Results from both arms were similar at baseline for each of the questionnaires.

Through the course of treatment, the median FACT-P QoL scores were greater with AAP vs. enzalutamide and were found to improve over time in the AAP arm, while remaining the same in the enzalutamide arm. A higher proportion of patients in the enzalutamide arm also had a worsening of their PHQ-9 depression scores and MoCA scores throughout the assessment.

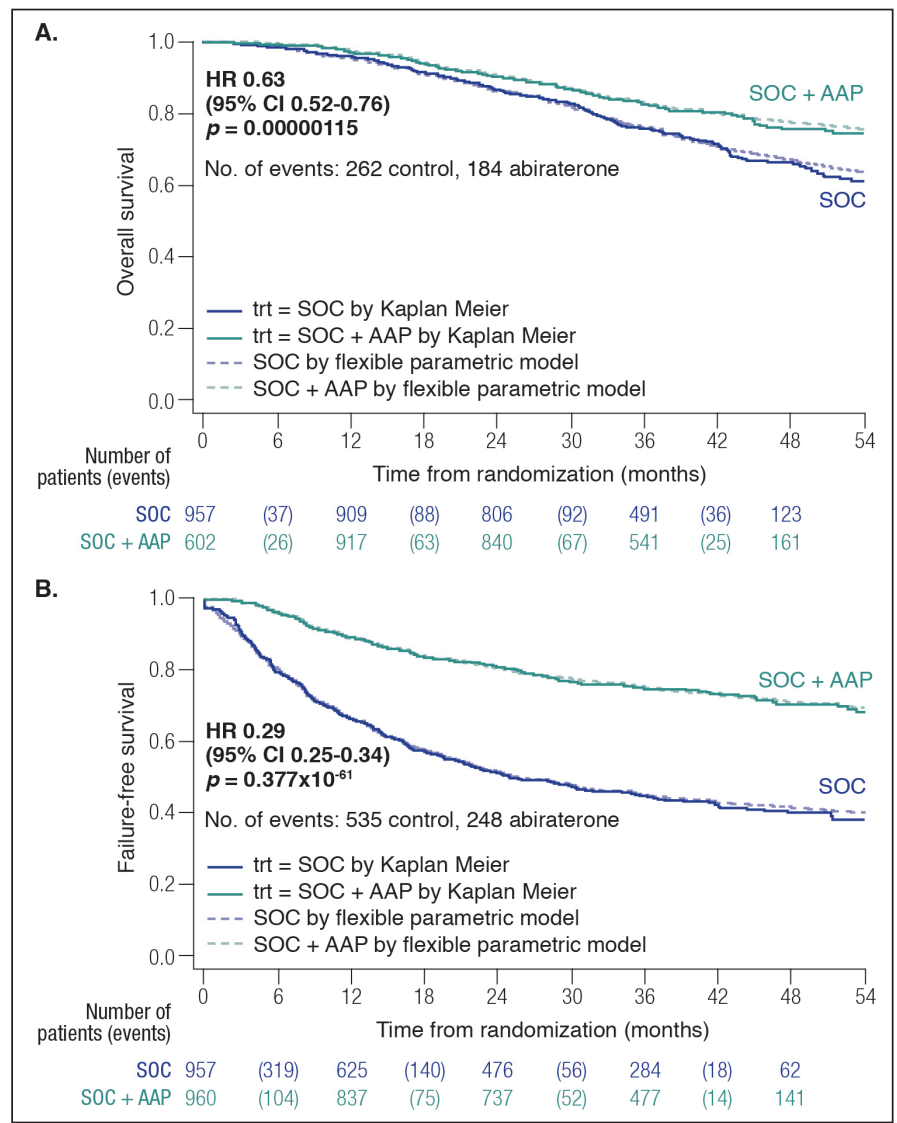

Fig 2. (A) Overall survival (OS); and (B) failure-free survival for abiraterone acetate $(A A)+$ prednisone $(P)+$ standard of care $(S O C)$ vs. SOC alone in highrisk prostate cancer (STAMPEDE). Cl: confidence interval; HR: hazard ratio.

These data, in combination with the first-line outcome data from this crossover study, suggest that AAP and enzalutamide have similar outcomes with respect to disease control, but that AAP has a better effect on QoL than treatment with enzalutamide.

\section{References}

1. Saad F, Chi KN, Finelli A, et al. The 2015 CUA-CUOG guidelines for the management of castration-resistant prostate cancer (CRPC). Can Urol Assoc J 2015;9:90-6. hittps://doi.org/10.5489/cuai.2526

2. Fizazi K, Tran N, Fein LE, et al. LATITUDE: A phase 3, double-blind, randomized trial of androgen-deprivation therapy with abiraterone acetate plus prednisone or placebos in newly diagnosed high-risk metastatic hormone-naïve prostate cancer. Oral presentation presented at ASCO 2017; abstract \#LB3.

3. James N, De Bono J, Spears MR, et al. Adding abiraterone for men with high-risk prostate cancer (PCa) starting long-term androgen-deprivation therapy (ADT): Survival results from STAMPEDE. Oral presentation presented at ASCO 2017; abstract \#LBO03.

4. Fizazi $\mathrm{K}$, Tran N, Fein L, et al. Abiraterone plus prednisone in metastatic, castration-sensitive prostate cancer. N Engl J Med 2017 Jun 4 [Epub ahead of print]. hittps://doi.org/10.1056/NEJMoal704174

5. James ND, de Bono IS, Spears MR, et al. Abiraterone for prostate cancer not previously treated with hormone therapy. N Engl J Med 2017 Jun 3 [Epub ahead of print]. htrps://doi.org/10.1056/NEJMoal702900

6. Chi KN, Annala M, Sunderland K, et al. A randomized phase 2 crossover study of abiraterone + prednisone (ABI) vs. enzalutamide (ENZ) for patients (pts) with metastatic, castration-resistant prostate cancer (mCRPC). Oral presentation presented at ASCO 2017; abstract \#5002.

7. Khalaf D, Sunderland K, Eigl B, et al. Assessment of quality of life (Q0L), cognitive function, and depression in a randomized phase 2 study of abiretarone acetate (ABI) plus prednisone (P) vs. enzalutamide (ENZA) for metastatic castrate-resistant prostate cancer (mCRPC). Poster presented at ASCO 2017; abstract \#5036. 